Document downloaded from:

http://hdl.handle.net/10251/100669

This paper must be cited as:

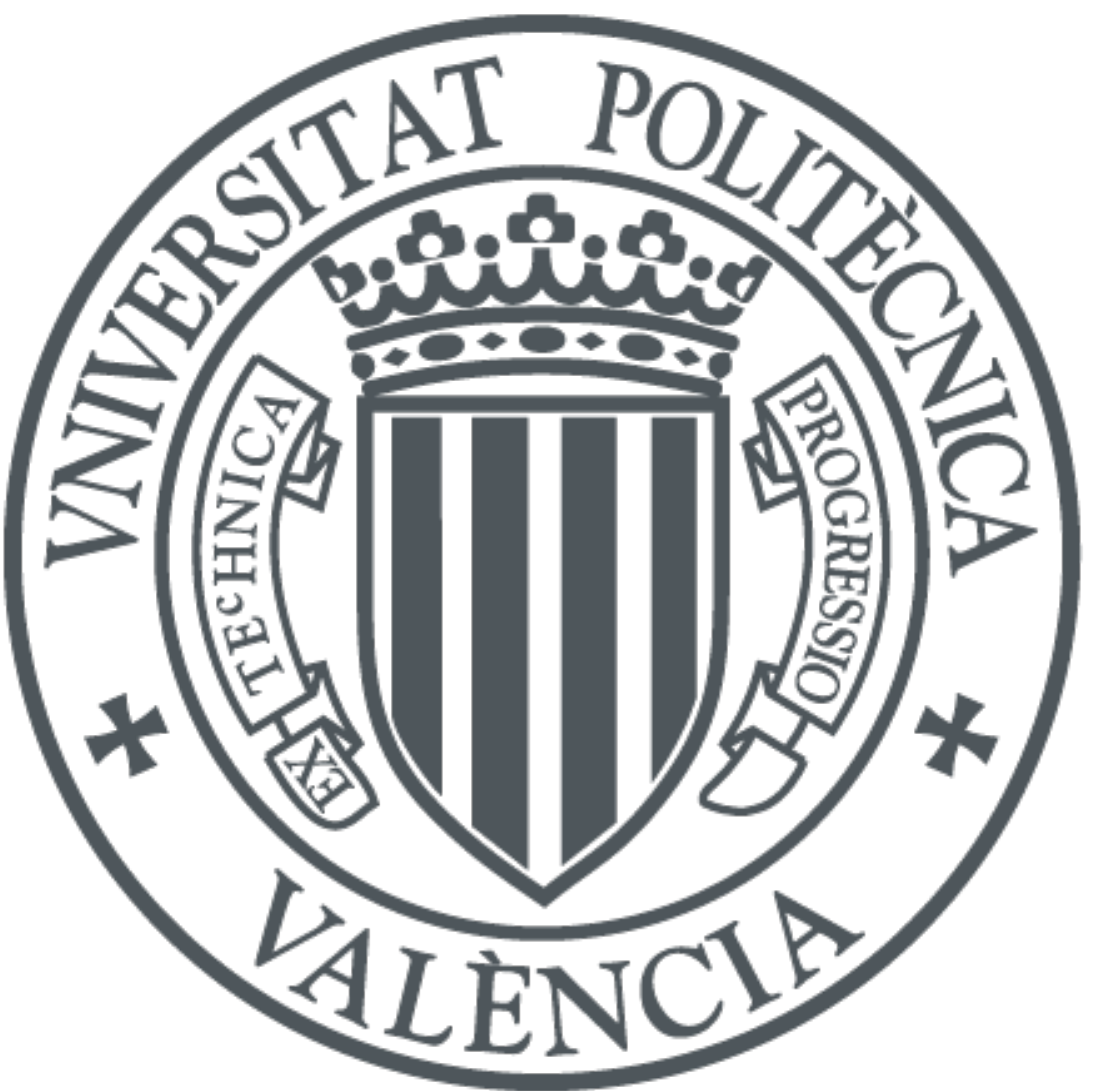

The final publication is available at https://doi.org/10.1063/1.1418423

Copyright American Institute of Physics

Additional Information 


\title{
Very large magnetoimpedance effect in FeCoNi ferromagnetic tubes with high order magnetic anisotropy
}

\author{
G. V. Kurlyandskaya, ${ }^{\text {a) }}$ H. Yakabchuk, and E. Kisker \\ Institut für Angewandte Physik, Heinrich Heine Universität Düsseldorf Universitätstraße 1, Bld.25.33.01.24, \\ D-40225, Düsseldorf, Germany \\ N. G. Bebenin \\ Institute of Metal Physics UD RAS Kovalevskaya strasse 18, GSP-170, 620219 Ekaterinburg Russia \\ H. García-Miquel \\ Departamento de Ing. Electrónica, Universidad Politécnica de Valencia, Camino de Vera, s/n, \\ 46022 Valencia, Spain \\ M. Vázquez \\ Instituto de Ciencia de Materiales, CSIC, Campus de Cantoblanco 28049 Cantoblanco (Madrid) Spain \\ V. O. Vas'kovskiy \\ Institute of Physics and Applied Mathematics, Ural State University, Lenin Ave. 51, 620083 Ekaterinburg, \\ Russia \\ ()
}

The extraordinarily high (up to $800 \%$ magnetoimpedance ratio) and sensitive magnetoimpedance effect has been found and studied in FeCoNi magnetic tubes electroplated onto $\mathrm{CuBe}$ nonmagnetic wire at frequency of about $1 \mathrm{MHz}$ order. Special annealing was done in order to induce magnetic anisotropy. The high harmonic response has been studied, and the harmonics show larger variations with the external magnetic fiel than the fundamental. This huge sensitivity of the harmonics (up to an order of the tens of thousands $\% / \mathrm{Oe}$ ) is promising in regard to the increase of the sensitivity of giant magnetoimpedance sensors. To explain the experiment results, we calculated the high frequency transverse susceptibility taking into account the magnetic anisotropy of firs and second orders. The susceptibility is extremely high at the points of orientational phase transitions in the magnetic layer which gives rise to strong nonlinear effects.

[DOI: $10.1063 / 1.1418423$ ]

\section{INTRODUCTION}

The magnetoimpedance (MI) phenomenon consists in the change of total impedance $Z$ of a ferromagnet under application of a magnetic fiel $H$. The phenomenon is classical in nature and in the case of a uniform sample results from the penetration depth dependence on the circular magnetic permeability. The general theory of the MI effect in a long cylinder was written long ago. ${ }^{1}$ Ordinarily, the effect is weak and therefore did not attract attention for many years after the experimental observation. ${ }^{2}$ The situation has been changed by Beach et al. ${ }^{3}$ and Panina et $a l^{4}{ }^{4}$ who reported a very large MI effect in amorphous wires with outstanding soft magnetic character. The very large - of the order of $100 \%$ - variation of $Z$ is now called the giant magnetoimpedance (GMI). The interest in GMI is due to possible applications in micromagnetic fiel detectors and magnetic recording heads. Since the MI depends on the permeability, which is sensitive to special treatments, there are many ways to modify the GMI responses. ${ }^{5-7}$

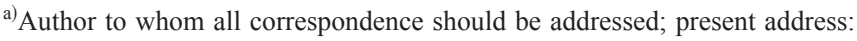
Universidad del País Vasco UPV-EHU, Facultad de Ciencias, Departamento de Electricidad y Electrónica, Apdo. 644, 48080 Bilbao, Spain; permanent address: Institute of Metal Physics UD RAS Kovalevskaya str. 18, GSP-170, 620219 Ekaterinburg, Russia; electronic mail: galina@polaris.we.lc.ehu.es
}

The GMI was observed not only in uniform wires ${ }^{8}$ and ribbons but also in magnetically coated nonmagnetic wires and layered structures. The electroplated $\mathrm{FeNi}, \mathrm{FeNiCo}$, and CoP wires studied in Refs. 9-13 are good examples. As a rule, the MI of a complex structure is higher than that of a uniform ferromagnet with the same magnetic characteristics. In contrast to the uniform materials, the change of the skin depth in a magnetic fiel can play no role in the GMI properties of the layered systems. ${ }^{9}$

Since the impedance is define as a ratio $U / I$ where $U$ is the voltage drop and $I$ is the ac current flowin through the sample, $Z$ can be introduced for the linear regime only. Some GMI experiments were performed however under nonlinear conditions. For example, the increase of MI with the increase of the ac current was reported in Refs. 9 and 14. Reference 15 investigated a sensor composed of a $\mathrm{Cu}$ wire sandwiched between Co-based amorphous ribbons, and found that the relative change in the output voltage at the exciting frequency $f_{\text {ex }}$ is much less than that at frequency $f=2 f_{\text {ex }}$.

In this work, we investigate the extraordinarily high and sensitive MI effect in FeCoNi magnetic tubes electroplated onto $\mathrm{CuBe}$ nonmagnetic wire in as-prepared samples as well as after fiel annealing. We study the changes in the voltage at frequencies corresponding to fundamental $F\left(f=f_{\text {ex }}\right)$, the firs $G 1\left(f=2 f_{\text {ex }}\right)$, and second $G 2\left(f=3 f_{\text {ex }}\right)$ harmonics. We 


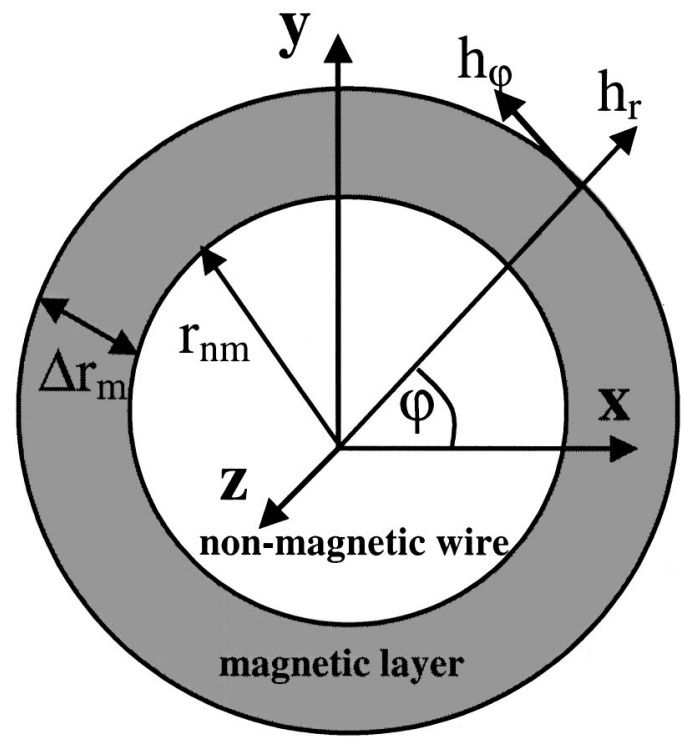

FIG. 1. The geometry of the nonmagnetic conductive wire of an axial symmetry covered by a magnetic layer. The external dc magnetic fiel and axis of the wire are parallel to $z$ axis. The ac magnetic fiel induced by exciting current is circumferential, i.e., only the azimuth component $h_{\varphi}(t)$ is not equal to zero.

reveal the connection between the intensity of the higher harmonics and the ac current. Our analysis shows that the strong GMI and the essential nonlinearity take place near a point of a spin-reorientation phase transition and are due to a very high sensitivity of the magnetic system in the vicinity of the transition points.

\section{THEORETICAL REMARKS}

Let us consider a long nonmagnetic cylinder of radius $r_{\mathrm{nm}}$ coated with a ferromagnetic layer of thickness $\Delta r_{m}$ (see Fig. 1). The coordinate system is chosen in such a way that the $z$ axis is along the axis of the cylinder. Our aim is to calculate the voltage $U(t)$ between two points (say, $z=0$ and $z=1)$ at the outer surface of the ferromagnetic layer if the total ac current $I(t)$ is given. Generally, the calculations are cumbersome, ${ }^{16}$ however, they become simple and straightforward if the layer is nonconducting and $\Delta r_{m} \ll r_{\mathrm{nm}} \cdot{ }^{9}$ Indeed, electric fiel $E(t)$ and magnetic induction $B(t)$ depend only on radius $r$ due to symmetry. Then, making use of Maxwell's equation $\sigma \times E=-(1 / c) \partial B / \partial t$, one obtains

$$
U(t)=Z_{\mathrm{nm}} I(t)+\frac{\Delta r_{m} l}{c} \frac{\partial B_{\varphi}}{\partial t},
$$

where $Z_{\mathrm{nm}}$ is the impedance of the nonmagnetic core, $B_{\varphi}$ is azimuthal component of the induction. In a linear regime, $B_{\varphi}(t)=B_{0 \varphi}+\mu_{\varphi \varphi} h_{\varphi}(t), \quad \mu_{\varphi \varphi}=1+4 \pi \chi_{\varphi \varphi}, \quad h_{\varphi}(t)$ is the magnetic fiel induced by the ac current: $h_{\varphi}(t)$ $=2 I(t) / c r_{\mathrm{nm}}$. Writing $I(t)=I \exp (-i \omega t), \quad U(t)=Z_{0} I(t)$ $+U_{1} \exp (-i \omega t)$ with $\omega=2 \pi f_{\text {ex }}$ we obtain

$$
Z=Z_{\mathrm{nm}}-\frac{2 i \omega \Delta r_{m} l}{c^{2} r_{\mathrm{nm}}} \mu_{\varphi \varphi} .
$$

At high frequency, displacements of the domain walls are of minor importance, so that one can calculate the magnetic susceptibility by making use of the Landau-Lifshitz equation

$$
\frac{\partial \mathbf{M}}{\partial t}=-\gamma\left[\mathbf{M}, \mathbf{H}^{\mathrm{eff}}\right]+\frac{G}{M_{s}}\left[\mathbf{M}, \frac{\partial \mathbf{M}}{\partial t}\right],
$$

where $\mathbf{M}$ is the magnetization, $|\mathbf{M}|$ is assumed to be equal to the saturation magnetization $M_{s}, \gamma$ is the gyromagnetic ratio, and $G$ stands for the damping constant, $\mathbf{H}^{\mathrm{eff}}=-\partial F / \partial \mathbf{M}$. The free energy $F$ can be written as

$$
F=F_{z}\left(M_{z}\right)+F_{r}\left(M_{r}\right)-\mathbf{H M},
$$

where $F_{z}$ describes the anisotropy along $z$ axes and $F_{r}$ - the anisotropy along the radius, including the shape anisotropy. Since $\Delta r_{m} \ll r_{\text {nm }}$, we may treat the magnetic cover as a thin layer and express $F_{r}$ as the addition of two terms

$$
F_{r}=2 \pi M_{r}^{2}-K_{r}\left(\frac{M_{r}}{M_{s}}\right)^{2}=-H_{a r} \frac{M_{r}^{2}}{M_{s}},
$$

where $M_{r}$ is the radial component of the magnetization and $H_{a r}$ is the radial component of the effective field As for $F_{z}$, we assume the expression $F_{z}=K_{1} \sin ^{2} \vartheta+K_{2} \sin ^{4} \theta$, where $\theta$ is the angle between the magnetization vector $\mathbf{M}$ and $z$ axis. The last term in this formula is usually omitted but we shall see next that the second order anisotropy can be essential. It is convenient to rewrite $F_{z}$ in the form

$$
F_{z}=-\left(H_{a 1}+2 H_{a 2}\right) M_{s} \xi^{2}+H_{a 2} M_{s} \xi^{4},
$$

where $H_{a 1,2}=K_{1,2} / M_{s}$ and $\xi=M_{z} / M_{s}=\cos \theta$. Let us firs consider the situation in a static magnetic fiel $\mathbf{H}_{0}$, which will be always assumed to be directed along the $z$ axis. The equilibrium magnetization, $\mathbf{M}_{0}$, corresponds to the minimal value(s) of free energy $F$. We shall take $H_{a r}<0$, so that $\mathbf{M}_{0}$ is perpendicular to the radius. The system can be in a collinear phase with $\xi= \pm 1$ or in an angle phase with $-1<\xi<+1$. In the latter case, the position of minimum obeys the equation $\partial F / \partial M_{0 z}=0$. If $H_{0 z}<2 H_{a 1}$, the $\xi=-1$ state is stable, and when $H_{0 z}>-2 H_{a 1}$, the $\xi=+1$ state is stable. The region of stability of the angle phase is given by inequality $-\left(H_{a 1}+2 H_{a 2}\right)+6 H_{a 2} \xi^{2}>0$ where $\xi$ obeys

$$
H_{0 z}+2\left(H_{a 1}+2 H_{a 2}\right) \xi-4 H_{a 2} \xi^{3}=0 .
$$

If $H_{0 z}=-\infty$, the system is in the $\xi=+1$ state. When $H_{0 z}$ is increased, the system passes from the $\xi=-1$ state to the $\xi=+1$ state by means of one or more magnetic phase transitions, see e.g. Ref. 1. For example, if $H_{a 2}=0$ and $H_{a 1}$ $>0$, there is the spin-reorientation transition of the firs order from the $\xi=-1$ to the $\xi=+1$ phase; on the contrary if $H_{a 2}$ $=0$ and $H_{a 1}<0$, there are two second-order transitions from the $\xi=-1$ phase to the angle phase and then from the angle phase to the $\xi=-1$ phase. If $H_{a 2} \neq 0$, there can be one transition of the firs order and another transition of the second order.

Now we can fin the susceptibility by solving the Landau-Lifshitz equation. After the standard procedure of linearization, we obtain:

(i) The magnetic layer is in a collinear state with $\xi= \pm 1$ : 


$$
\chi_{\varphi \varphi}=\frac{M_{s}\left( \pm H_{0 z}+2 H_{a 1}-2 H_{a r}-i G \frac{\omega}{\gamma}\right)}{-\left(\frac{\omega}{\gamma}\right)^{2}+\left( \pm H_{0 z}+2 H_{a 1}-i G \frac{\omega}{\gamma}\right)\left( \pm H_{0 z}+2 H_{a 1}-2 H_{a r}-i G \frac{\omega}{\gamma}\right)} .
$$

This is obviously the susceptibility of a thin ferromagnetic layer in magnetic fiel $\pm H_{0 z}+2 H_{a 1}$.

(ii) The magnetic layer is in the angle phase,

$$
\chi_{\varphi \varphi}=\frac{M_{s} \xi^{2}\left(-2 H_{a r}-i G \frac{\omega}{\gamma}\right)}{-\left(\frac{\omega}{\gamma}\right)^{2}+\left[-2 H_{a r}-i G \frac{\omega}{\gamma}\right]\left[\left(-2\left(H_{a 1}+2 H_{a 2}\right)+12 H_{a 2} \xi^{2}\right)\left(1-\xi^{2}\right)-i G \frac{\omega}{\gamma}\right]} .
$$

In addition to intrinsic damping, which is described by the constant $G$, there can be the frequency independent broadening due to magnetic inhomogeneities. We do not write this term explicitly because it is not our aim here to analyze the linewidth broadening in detail. The discussion on homogeneous and inhomogeneous linewidth can be found in a recent review. ${ }^{17}$

For the linear regime to exist and hence Eqs. (2), (8), and (9) to be applicable, the inequality $\chi_{\varphi \varphi} h_{\varphi} \ll M_{S}$ must be fulfilled If it is not the case, we have to write $B_{\varphi}(t)=B_{0 \varphi}+\sum_{k=1}^{\infty} B_{\varphi k} \exp (-i k \omega t), \quad U(t)=Z_{\mathrm{nm}} I \exp (-i \omega t)$ $+\sum_{k=1}^{\infty} U_{k} \exp (-i k \omega t)$. To fin the relations between $I, B_{\varphi k}$, and $U_{k}$, one has to solve the Landau-Lifshitz equation at any magnetic field including the vicinity of all spinreorientation phase transitions. This is far beyond the scope of the present article. It is worth, however, noting that from Eq. (1) one can readily derive $U_{k} / U_{1}=k B_{\varphi k} / B_{\varphi 1}$, therefore, the $k \geqslant 2$ harmonics are more pronounced in $U(t)$ than in $B(t)$.

It is also noteworthy that the term "impedance" belongs to the linear response theory. Nevertheless, for brevity and following the tradition of the GMI studies, we shall use this term for the voltage/current ratio throughout the article regardless of degree of the nonlinearity.

\section{EXPERIMENTAL DETAILS}

Our sample is a $100 \mu \mathrm{m}$ diameter nonmagnetic $\mathrm{CuBe}$ wire coated by a $1 \mu$ m-thick electroplated $\mathrm{Fe}_{20} \mathrm{Co}_{6} \mathrm{Ni}_{74}$ layer. Before the deposition of the magnetic layer, the pure $\mathrm{Cu}$ of 3-4 $\mu \mathrm{m}$ was deposited onto $\mathrm{CuBe}$ wire in order to reduce the roughness of the $\mathrm{CuBe}$ substrate. Resistivity of the magnetic coating was about $3.9 \mu \Omega \mathrm{cm}$ and that of the magnetic layer about $15 \mu \Omega$. The saturation magnetization of the magnetic layer was $M_{s} \approx 900$ Oe at room temperature. A current flowin along the wire during the electrodeposition causes the circumferencial component of the magnetization due to the presence of the circular magnetic field The length of the samples used for all measurements was $60 \mathrm{~mm}$. The longitudinal hysteresis loops of the samples were measured by conventional inductive fluxmetri method at $50 \mathrm{~Hz}$ frequency in a magnetic fiel applied parallel to the wire axis. Field annealing was done in inert $\mathrm{Ar}$ atmosphere under ac fiel applied along the axis of the wire for $1 \mathrm{~h}$ at $320^{\circ} \mathrm{C}$ and then cooling in the magnetic dc fiel of 20 Oe.

We have developed the measuring system with the possibility of the measurements of the voltage drop across the sample as a function of the external fiel using standard fourpoint method. The variable magnetic fiel was generated by a pair of Helmholtz coils. The maximum magnetic fiel for these measurements was $H_{\max }= \pm 32 \mathrm{Oe}$. At every magnetic field the exciting current amplitude was measured using a high frequency current transformer (to avoid undesirable ground in the circuit) and the output of the function generator was changed accordingly in order to maintain the constant current amplitude regardless of the voltage variations. Therefore, the experimentally measured voltage was proportional to the total impedance $Z$. The MI was measured in the frequency region of $1-10 \mathrm{MHz}$, for ac sinusoidal driving currents of $I_{\mathrm{rms}}=35 \mathrm{~mA}$ or $10 \mathrm{~mA}$ both in increasing and decreasing external fiel ("up" MI curve for increasing and "down" for decreasing magnetic fiel $)$. The MI ratio is define as $\Delta Z / Z(H)=100 x\left[Z(H)-Z\left(H_{\max }\right)\right] / Z\left(H_{\max }\right)$.

The distorted shape of the output signal indicated the nonlinear behavior and the generation of high harmonics. We measured the dependence of the Fourier components at the fundamental $F\left(f=f_{\text {ex }}\right)$, the firs $G 1\left(f=2 f_{\text {ex }}\right)$, and second $G 2\left(f=3 f_{\text {ex }}\right)$ harmonics in the external magnetic fiel applied parallel to the wire axis.

\section{RESULTS OF MEASUREMENTS}

The effect of annealing is shown in Fig. 2. The annealing reduces the saturation fiel but does not change the coercive force as it is seen in Fig. 2(a). Figure 2(b) shows the $\Delta Z / Z$ curves of the samples in increasing magnetic fields One can see the broad maximum at a negative fiel and the jump in a positive field Unlike the $\Delta Z / Z$ curves, the hysteresis loops of the samples have no visible peculiarities in the negative fields

The maximum value of the MI ratio depends on frequency of the driving current. Figure 3 shows that the MI of the as-prepared samples grows quickly with frequency until $2 \mathrm{MHz}$, and then becomes smaller. The MI ratio of the annealed wire increases until $1 \mathrm{MHz}$ and almost does not depend on the frequency at higher frequencies. The as-prepared sample shows a very high $\Delta Z / Z$ ratio of about $800 \%$ for 1.5 

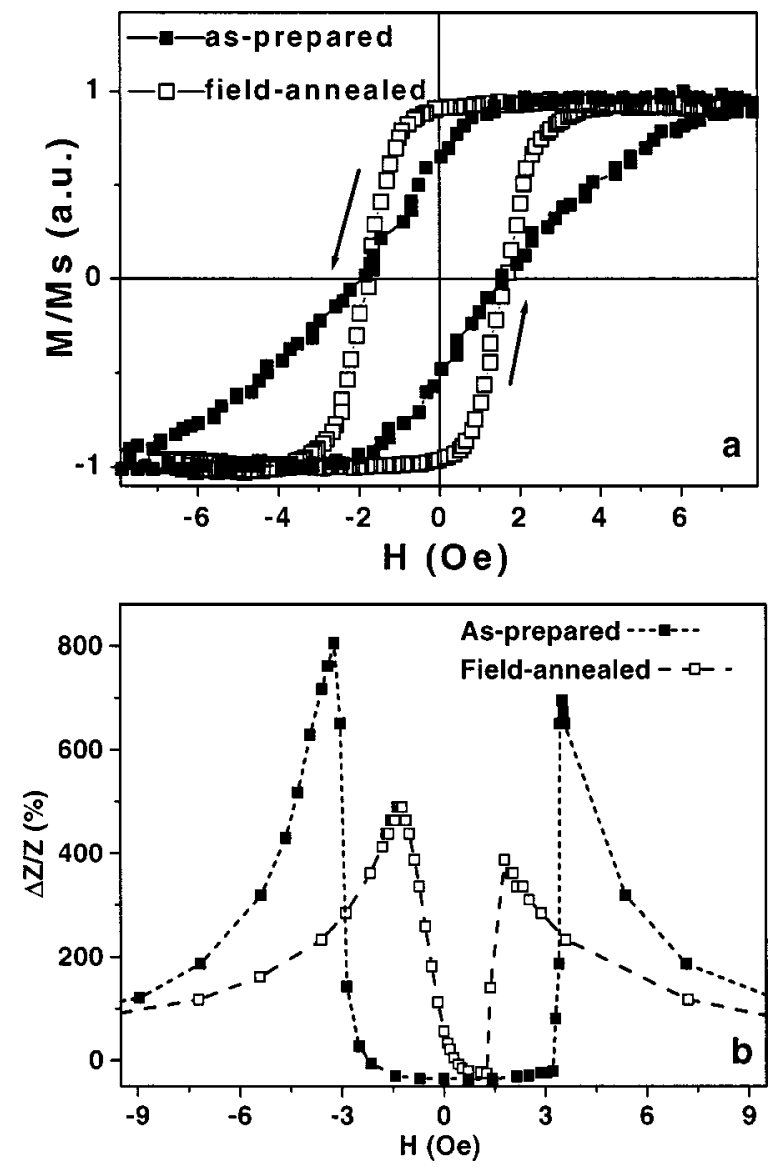

FIG. 2. Longitudinal hysteresis loops of FeCoNi electroplated wires; (a) GMI responses of $\mathrm{FeCoNi}$ electroplated wires in increasing fiel at a frequency of $1.5 \mathrm{MHz}$.

$\mathrm{MHz}$, which is higher than $\Delta Z / Z$ ratio of the field-anneale sample (maximum of about $500 \%$ ). However if $f_{\text {ex }}>5 \mathrm{MHz}$, the MI of the field-anneale sample is higher than that of the as-prepared sample. The weak dependence of the $\Delta Z / Z$ ratio of the field-anneale wire in the wide range of the frequencies can be important for technological applications.

To understand the origin of such a high MI effect, we made the Fourier analysis of the output voltage at the fre-

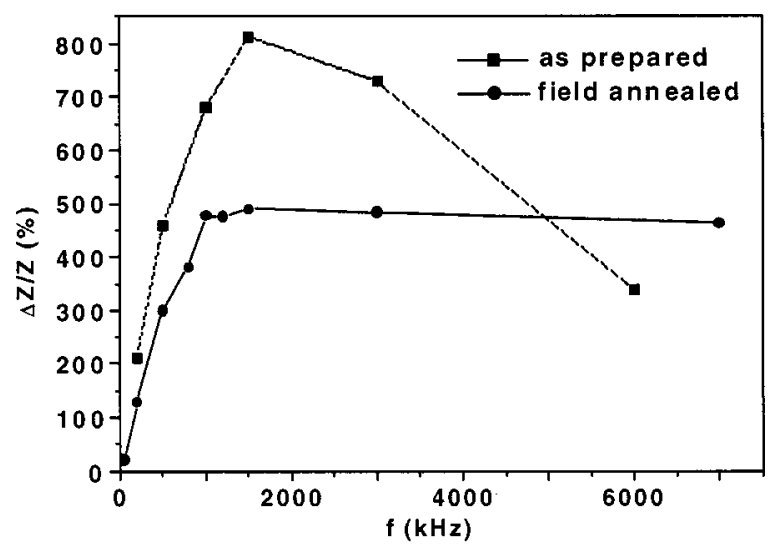

FIG. 3. Frequency dependence of the maximum of the MI ratio $\Delta Z / Z$ of the $\mathrm{FeCoNi}$ electroplated wires.
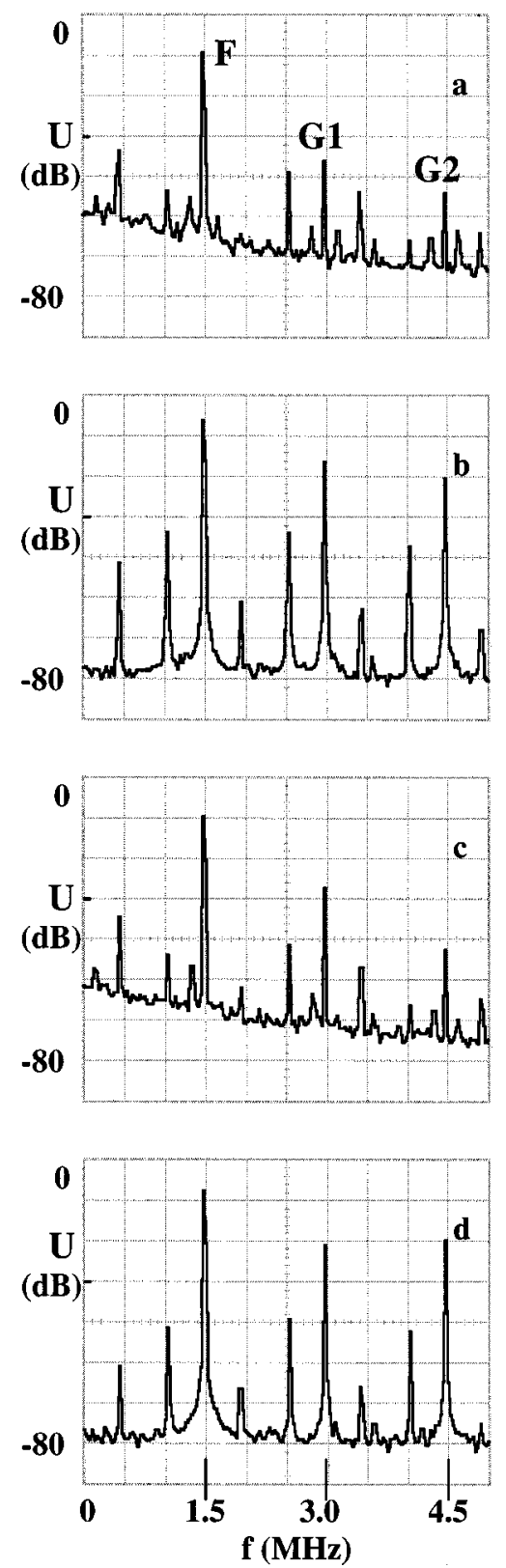

FIG. 4. Fourier analysis of the voltage $U(t)$ induced across the FeCoNi electroplated fiel annealed wire. The frequency of the drive current was 1.5 $\mathrm{MHz}$ and the intensity of the ac current of $I_{\mathrm{rms}}=35 \mathrm{~mA}$. Axial field were: (a) -32 Oe; (b) -1.5 Oe; (c) +1.2 Oe; and (d) +2.2 Oe. Three peaks at fundamental, $F$, (frequency is equal to the frequency, $f_{\mathrm{ex}}=1.5 \mathrm{MHz}$, of the exiting signal of the generator), the first $G 1$, (at a frequency $2 f_{\mathrm{ex}}=3 \mathrm{MHz}$ ) and the second, $G 2$, (at a frequency $3 f_{\mathrm{ex}}=4.5 \mathrm{MHz}$ ) are of interest to us.

quency of $1.5 \mathrm{MHz}$ where the highest MI effect is observed. Figure 4 shows the Fourier analysis of the experimental voltages of the field-anneale sample for the measurements in these states: Fig. 4(a) — magnetic saturated state, Fig. 4(b) negative fiel near the firs MI maximum, Fig. 4(c) - small positive fiel corresponding to the minimum of the MI ratio, and Fig. 4(d) - the second MI maximum in a positive field The generation of high harmonics near the field of GMI maxima [Figs. 4(b) and (d)] is clearly seen. Figure 5 represents the fiel dependencies of the $F, G 1$, and $G 2$ voltages taken from the Fourier analysis. In the saturated state, the $G 1$ 

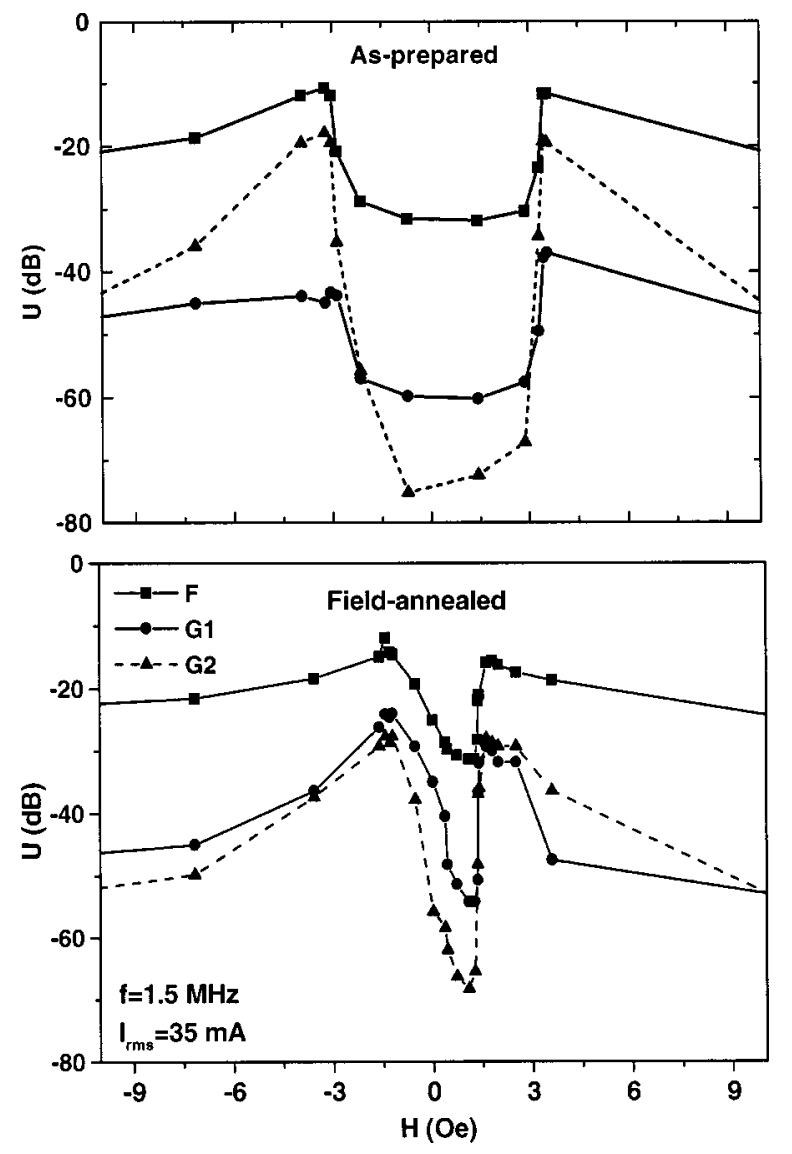

FIG. 5. The external fiel dependence of the Fourier components of the voltages of the FeCoNi electroplated wires. The voltage axis is in the logarithm scale. The voltages at fundamental, $F$, (frequency is equal to the frequency, $f_{\mathrm{ex}}=1.5 \mathrm{MHz}$, of the exiting signal of the generator), the first $G 1$, (at a frequency $2 f_{\mathrm{ex}}=3 \mathrm{MHz}$ ) and the second, $G 2$, (at a frequency $3 f_{\mathrm{ex}}=4.5 \mathrm{MHz}$ ) harmonics are of interest to us.

and $G 2$ amplitudes are much smaller than the $F 1$ amplitude for both the samples but near the field corresponding to the MI maximum (states $b$ and $c$ ) the high harmonic generation is clearly seen especially for the as-prepared sample. In a high field the $G 1$ and $G 2$ harmonics amplitudes are very small. The relative changes of $G 1$ and $G 2$ in the fiel between the $a$ and $b$ states, and especially between $b$ and $c$, are higher than those at the fundamental frequency.

For each harmonic, we have calculated the induced voltage in decimal scale (Fig. 6) and define $\Delta U / U$ $=100 x\left[U(H)-U\left(H_{\max }\right)\right] / U\left(H_{\max }\right)$ with $H_{\max }=32$ Oe. Calculating the sensitivity as a change of $\Delta U / U$ per unit of the applied field we have obtained the maximum voltage changes of $8 \times 10^{4} \% / \mathrm{Oe}$ for the $G 2$ harmonic, 3.7 $\times 10^{2} \% / \mathrm{Oe}$ for $G 1$, and $3.8 \times 10^{2 \%} / \mathrm{Oe}$ at a fundamental frequency in the as-prepared sample showing the highest GMI effect. This means the difference of the sensitivity of 21 times between $F$ and $G 2$, and similar sensitivity for $F$ and $G 1$. We obtained the maximum voltage sensitivity of 3 $\times 10^{4} \% / \mathrm{Oe}$ for $G 2$ harmonic, $6 \times 10^{3} \% / \mathrm{Oe}$ for $G 1$, and $1.2 \times 10^{3} \% / \mathrm{Oe}$ at a fundamental frequency in field-anneale wire. This shows a difference of sensitivity of 25 times between $F$ and $G 2$ and 5 times for $F$ and $G 1$. In a previous
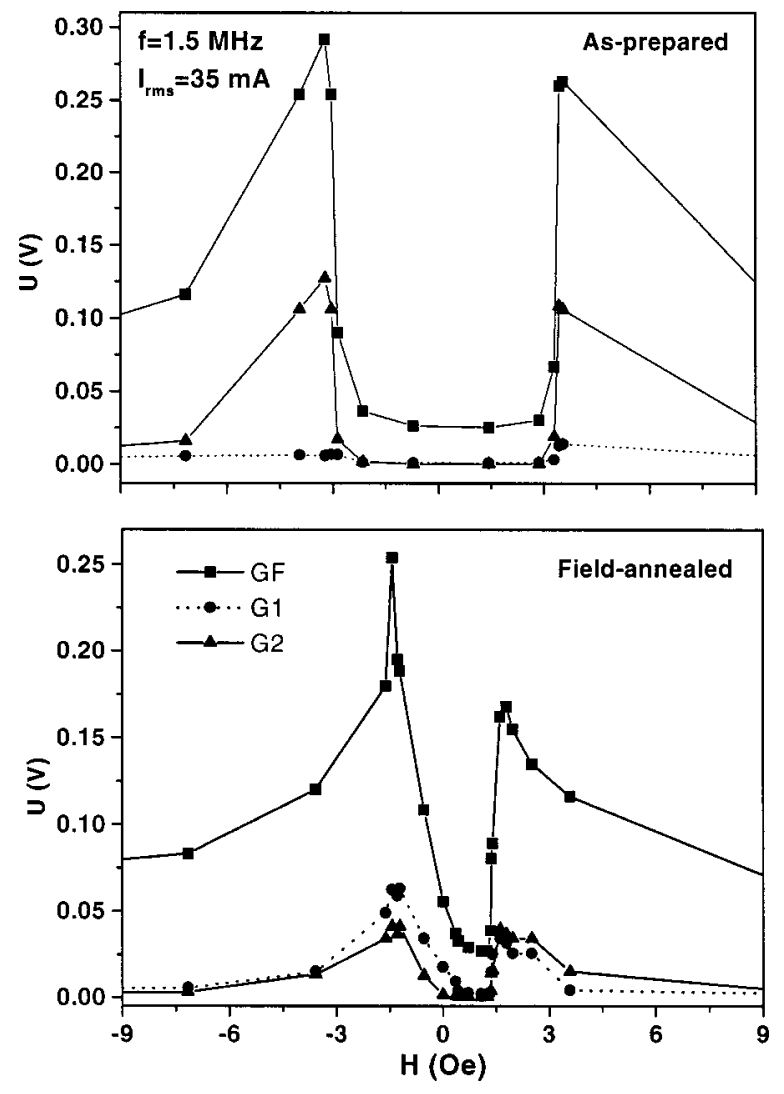

FIG. 6. The external fiel dependence of the Fourier components of the voltages of the FeCoNi electroplated wires. The voltage axis is in the decimal scale. The voltages at fundamental, $F$, (frequency is equal to the frequency, $f_{\mathrm{ex}}=1.5 \mathrm{MHz}$, of the exiting signal of the generator), the first $G 1$, and the second, $G 2$, harmonics are of interest to us.

work, ${ }^{15}$ a difference of 16 times for the sensitivities of 9.8\%/Oe for $F$ and 151\%/Oe for $G 1$ (G2 was not measured) was reported.

Figure 7 shows the dependence of the MI ratio of the as-prepared wire on the driving current value. The maximum MI ratio at the current of $10 \mathrm{~mA}$ is only $160 \%$ at the frequency of $1.5 \mathrm{MHz}$ but the increase of the current intensity up to $35 \mathrm{~mA}$ results in $800 \%$ MI ratio at the same frequency. The $G 2$ harmonic exhibits an even more dramatic change. The $G 2$ variation at the current of $10 \mathrm{~mA}$ is negligible while the change at the ac current amplitude of $35 \mathrm{~mA}$ is very large. If the current is small, only the change in the $F$ harmonic is essential, but for the higher current, the $G 2$ harmonic changes significantl .

\section{DISCUSSION}

The experimental results of the preceeding section show that the extremely high MI is due to nonlinear processes in the magnetic layer. The developed nonlinearity arises, however, only in the vicinity of special values of $H_{0 z}$, which do not necessarily correspond to visible peculiarities at the longitudinal hysteresis loop. Our aim now is to discuss the origin of the nonlinearity. We shall consider the field-anneale sample because this sample exhibits the maximum of mag- 


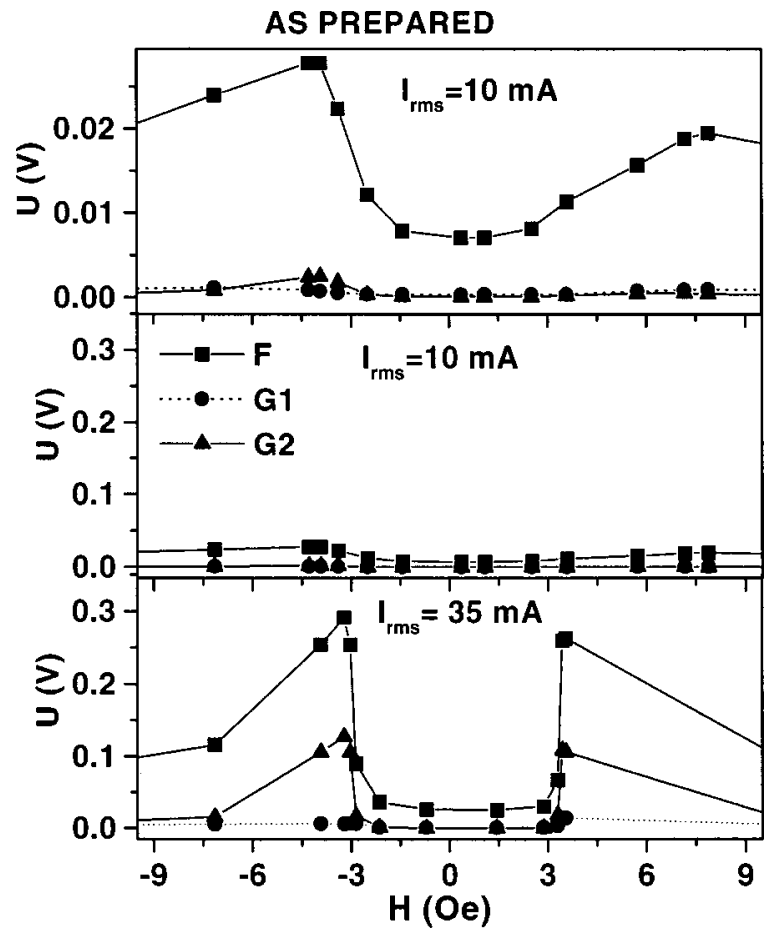

FIG. 7. The external fiel dependence of the Fourier components of the voltage of the as-prepared $\mathrm{FeCoNi}$ electroplated wire for the exiting current of 10 and $35 \mathrm{~mA}$. The voltage axis is in the decimal scale. The voltages at fundamental, $F$, (frequency is equal to the frequency, $f_{\mathrm{ex}}=1.5 \mathrm{MHz}$, of the exiting signal of the generator), the first $G 1$, (at a frequency $2 f_{\mathrm{ex}}=3 \mathrm{MHz}$ ) and the second, $G 2$, (at a frequency $3 f_{\text {ex }}=4.5 \mathrm{MHz}$ ) harmonic are of interest to us. The second harmonic $G 2$ shows huge variations in the fiel near the GMI maximum for the current of $35 \mathrm{~mA}$ and very small changes for the current of $10 \mathrm{~mA}$.

netoimpedance in lower field than the as-prepared sample and no frequency dependence in the wide frequency range.

Let us examine the $\Delta Z / Z$ curve shown in Fig. 2. One can see the broad maximum at $H_{0 z 1}=-1.25 \mathrm{Oe}$ and the jump at $H_{0 z 2}=+1.25 \mathrm{Oe}$. We can assume that there is the second-order phase transition between the $\xi=-1$ phase and the angle phase at $H_{0 z 1}$ and the first-orde transition at $H_{0 z 2}$. One can show easily that the second-order transition occurs at $H_{0 z}=-2 H_{a 1}$, so that $H_{a 1}=-0.63 \mathrm{Oe}$. It can be shown that the first-orde transition from the angle phase to the $\xi=-1$ phase occurs at $H_{0 z}=4 / 3\left(H_{a 1}+2 H_{a 2}\right)^{3 / 2} /\left(6 H_{a 2}\right)^{1 / 2}$, so that we obtain $H_{a 2}=1.25 \mathrm{Oe}$.

Now we are able to calculate the transverse magnetic susceptibility $\chi_{\varphi \varphi}$ by making use of Eqs. (8) and (9). The saturation magnetization $M_{s}$ is equal to $900 \mathrm{G}$. The anisotropy constant $K_{r}$ is unknown, and we take $K_{r}=0$; then $-2 H_{a r}=4 \pi M_{s}$. For a single crystal, the damping constant is usually of the order of 0.1 or less, but for our polycrystalline layer, we have taken $G=1$. The result of calculations of susceptibility for increasing $H_{0}$ is shown in Fig. 8. The most interesting one is the $\operatorname{Im}\left(\chi_{\varphi \varphi}\right)-H_{0 z}$ curve because $\operatorname{Im}\left(\chi_{\varphi \varphi}\right)$ is proportional to the energy absorbed by the magnetic layer. The curve has two peaks corresponding to two orientation phase transitions. The peaks differ in shape: the firs (in the negative fiel ) is broad and shows no special features, which reflect the fact that the transition at the fiel is of the second
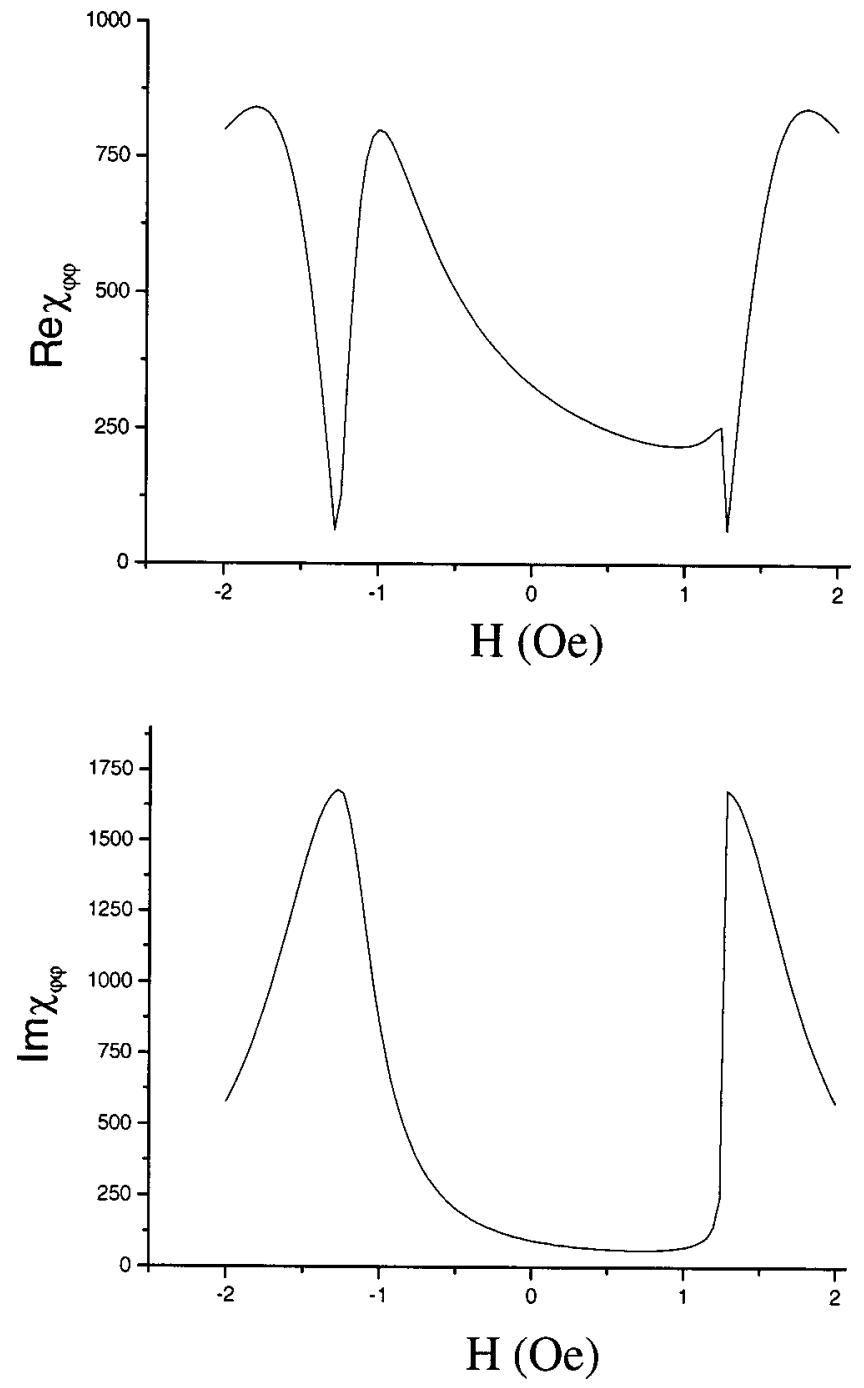

FIG. 8. The results of the model calculations. The $\operatorname{Im}\left(\chi_{z \varphi}\right)-H_{0}$ and $\operatorname{Re}\left(\chi_{z \varphi}\right)-H_{0}$ curves have very sharp extremes at $1.2 \mathrm{Oe}$, which originate in the first-orde transition. The $\operatorname{Im}\left(\chi_{\varphi \varphi}\right)-H_{0}$ curve has two peaks due to two orientation phase transitions. The peaks differ in shape: the firs (in the positive fiel ) is broad and shows no special features, which reflect the fact that the transition at the fiel is of the second order, the second peak (in the negative fiel ) shows a jump in the susceptibility value because the transition at the negative fiel is of the firs order; $H=\mathbf{H}_{\mathbf{0}} .85$

order; to the contrary, the second peak (in the positive fiel ) shows a jump in the susceptibility value because the transition at the positive fiel is of the firs order. The difference between the transitions is also clear on the $\operatorname{Re}\left(\chi_{\varphi \varphi}\right)-H_{0 z}$ curve. It is to be noted that the static longitudinal susceptibility $\chi=d M_{0 z} / d H_{0 z}$ that can be found by differentiating the hysteresis loop has no peculiarity at the fiel at which the transition between the $\xi=-1$ and the angle phases occurs, which agrees with the experiment.

The maximum $\chi_{\varphi \varphi}$ is of the order of $10^{3}$. Since the magnetic fiel $h_{\varphi}$ is of the order of $1 \mathrm{Oe}$, the inequality $h_{\varphi}$ $\ll M_{s}$ is not fulfille near the transition points and nonlinear effects must arise. Beyond the close vicinity of $H_{0 z 1}$ or $H_{0 z 2}$, the susceptibility is of the order of $10^{2}$ or less and the inequality $h_{\varphi} \ll M_{s}$ holds, which determines the linear regime. These conclusions agree with experiments completely, 
therefore, the developed nonlinearity in the vicinity of $H_{0 z 1}$ or $H_{0 z 2}$ are due to the high sensitivity of magnetic system at the points of spin-reorientation phase transitions.

\section{CONCLUSIONS}

The $\mathrm{Fe}_{20} \mathrm{Co}_{6} \mathrm{Ni}_{74}$ magnetic layer, $1 \mu \mathrm{m}$ thick, has been electrodeposited onto nonmagnetic $\mathrm{CuBe}$ wire. Extraordinarily high (up to $800 \%$ ) MI ratio and sensitivity (up to 4500 $\% / \mathrm{Oe}$ ) are observed at frequencies about $1 \mathrm{MHz}$.

Strong nonlinear effects have been found and studied in detail. Firstly, the high harmonic generation has been investigated. The second and the firs harmonic show much larger variation with the external magnetic fiel than the fundamental one. The huge sensitivity of the harmonics is promising from the viewpoint of applications. The strong dependence of the GMI ratio on the intensity of the exiting current confirm the role of the nonlinearity in formation of the very high GMI ratio.

Our model calculations have shown that such a behavior is due to the very high sensitivity of the magnetic system to the circumferential magnetic field induced by the ac current, in the vicinity of the spin-reorientation phase transitions occurring in the magnetic layer. The magnetic anisotropy of a higher order can play a key role and generally can not be ignored. In short, very high MI has an essentially nonlinear effect existing in the vicinity of a spin-reorientation phase transition.

\section{ACKNOWLEDGMENTS}

One of the authors (G.V.K.) wants to acknowledge all of the staff of the Institut für Angewandte Physik Heinrich
Heine Universität Düsseldorf for thorough support during her stay. The authors thank Stefan Mandela, Claudius Möhrle, and Ulrich Rosowski (Institut für Angewandte Physik) for excellent technical support. Another author (N.G.B.) was supported in part by RFBR Grant No. 00-1596745.

${ }^{1}$ L. D. Landau and E. M. Lifshitz, Electrodynamics of Continuous Media (Pergamon, Oxford, 1975), p. 195.

${ }^{2}$ E. P. Harrison, G. L. Turney, H. Rowe, and H. Gollop, Proc. R. Soc. Edinburgh 157, 651 (1937).

${ }^{3}$ R. S. Beach and A. E. Berkowitz, Appl. Phys. Lett. 64, 3652 (1994).

${ }^{4}$ L. V. Panina and K. Mohri, Appl. Phys. Lett. 65, 1189 (1994).

${ }^{5}$ R. L. Sommer and C. L. Chien, J. Appl. Phys. 79, 5139 (1996).

${ }^{6}$ J. P. Sinnecker, P. Tiberto, G. V. Kurlyandskaya, E. H. C. P. Sinnecker, M. Vázquez, and A. Hernando, J. Appl. Phys. 84, 5814 (1998).

${ }^{7}$ G. V. Kurlyandskaya, J. M. Garcia-Beneytez, M. Vázquez, J. P. Sinnecker, V. A. Lukshina, and A. P. Potapov, J. Appl. Phys. 83, 6581 (1998).

${ }^{8}$ N. A. Usov, A. S. Antonov, and A. N. Lagar'kov, J. Magn. Magn. Mater. 185, 159 (1998)

${ }^{9}$ R. S. Beach, N. Smith, C. L. Platt, F. Jeffers, and A. E. Berkowitz, Appl. Phys. Lett. 68, 2753 (1996)

${ }^{10}$ D. García, G. V. Kurlyandskaya, M. Vázquez, F. I. Toth, and L. K. Varga, J. Magn. Magn. Mater. 203, 208 (1999).

${ }^{11}$ G. V. Kurlyandskaya, J. M. Barandiarán, J. Gutierrez, D. García, M. Vázquez, and V. O. Vas'kovskiy, J. Appl. Phys. 85, 5438 (1999).

${ }^{12}$ G. V. Kurlyandskaya, J. M. Barandiarán, J. L. Muñoz, J. Gutierrez, D. Garcia, M. Vázquez, and V. O. Vas'kovskiy, J. Appl. Phys. 87, 4822 (2000).

${ }^{13}$ J. M. Garcia, A. Asenjo, J. P. Sinnecker, and M. Vazquez, J. Magn. Magn. Mater. 215, 352 (2000).

${ }^{14}$ A. Yelon, M. Britel, D. Menard, and P. Ciureanu, Physica A 241, 439 (1997).

${ }^{15}$ S. Iida, O. Ishii, and S. Kambe, Jpn. J. Appl. Phys., Part 2 37, L869 (1998).

${ }^{16}$ A. Gromov and V. Korenivski, J. Phys. D 33, 773 (2000).

${ }^{17}$ M. Farle, Rep. Prog. Phys. 61, 755 (1998). 